\section{Eficacia de un programa educativo estructurado en población diabética chilena}

\author{
ROBERTO BÄCHLER ${ }^{1}$, VERÓNICA MUJICA ${ }^{2}$, \\ CAROLINA ORELLANA ${ }^{1, a}$, DIANA CÁCERES ${ }^{3}$, NANCY CARRASCO ${ }^{4, b}$, \\ CAROL DAVIDSON ${ }^{5, b}$, ANA LUISA JIMÉNEZ ${ }^{6, b}$, PATRICIA LUNA ${ }^{3, b}$, \\ EVELYN VALLADARES ${ }^{3, b}$, ALEJANDRA VERGARA $^{3, b}$
}

\section{Effectiveness of a structured educative program in Chilean diabetic patients}

Background: Structured educative programs have demonstrated their usefulness as a strategy to improve metabolic control in diabetic patients. Aim: To evaluate the effectiveness of a structured educative program for Chilean diabetic patients. Material and Methods: A randomized clinical trial in diabetic patients with glycosylated hemoglobin over $7.5 \%$. One hundred fifteen patients were studied, 59 patients participated in the structured educative program (experimental group) and 56 patients received no structured education (control group). Patients were followed for 12 months. Results: Between baseline and 12 months of follow-up, glycosylated hemoglobin changed from 10.05 to $9.11 \%$ in experimental patients and from 9.86 to $9.25 \%$ in controls. No significant differences between experimental and control groups in other clinical and metabolic parameters were observed. In the experimental group, glycosylated hemoglobin reductions differed among the different educators who carried out the program. Conclusions: A structured educative program resulted in a 35\% greater reduction in glycosylated hemoglobin levels, compared with a control group. Metabolic control improvement differed between the educators who carried out the program.

(Rev Med Chile 2017; 145: 181-187)

Key words: Diabetes Mellitus; Education; Health Education; Public Health.

\author{
'Departamento Información para \\ la Gestión. Dirección Servicio \\ Salud Maule. Talca, Chile. \\ ${ }^{2}$ Programa de Salud \\ Cardiovascular. Dirección Servicio \\ Salud Maule. Talca, Chile. \\ ${ }^{3}$ Centro de Salud Familiar Ararú \\ Méndez. Departamento de Salud \\ Municipal Parral. Parral, Chile. \\ 4. Posta de Salud Rural Mercedes. \\ Departamento de Salud \\ Municipal Talca. Talca, Chile. \\ 5. Centro de Salud Municipal \\ Curicó. Departamento de Salud \\ Municipal Curicó. Curicó, Chile. \\ 6. Centro de Salud Municipal Dr. \\ José Dionisio Astaburuaga. Talca, \\ Chile. \\ angeniero Estadístico. \\ bEnfermera Universitaria. \\ El material audiovisual del \\ Programa Educativo para \\ Pacientes Diabéticos fue \\ financiado por el Servicio de \\ Salud Maule. \\ Recibido el 9 de agosto de 2016, \\ aceptado el 13 de enero de \\ 2017. \\ Correspondencia a: \\ Roberto Bächler \\ Dirección de Servicio Salud \\ Maule, 1 Norte $\mathrm{N}^{\circ} 9634^{\circ}$ Piso. \\ Oficina 401. Talca, Chile \\ Fono 71-2411696 \\ rbachler@ssmaule.cl
}

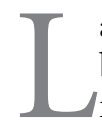

a diabetes mellitus es una enfermedad metabólica crónica, no transmisible y de etiología multifactorial producida por defectos en la secreción y/o acción de la insulina; entre 90 y 95\% de los sujetos afectados por esta patología presentan una diabetes mellitus tipo 2. La prevalencia de diabetes mellitus en Chile, según la encuesta Nacional de Salud del año 2010, es de 9,4\% (IC 8,1-10,8) en la población mayor de 17 años $^{1}$. La evidencia científica disponible, particularmente el estudio United Kingdom Prospective Diabetes Study (UKPDS), ha demostrado que los pacientes diabéticos que no tienen un adecuado control de su afección incrementan entre 3 y 10 veces los riesgos de sufrir complicaciones macrovasculares -enfermedad cerebro vascular, infarto al miocardio, enfermedad vascular periférica- y/o microvasculares-retinopatía, nefropatía, neuropatía-2,3. A nivel mundial, existe consenso respecto a que la mejor práctica para disminuir la frecuencia de complicaciones es que el paciente se haga responsable del cuidado de su enfermedad; la estrategia utilizada para que el enfermo asuma un rol protagónico en el control de su afección es la educación $\mathrm{n}^{4-21}$. La revisión sistemática realizada por Deakin $\mathrm{T}$ et al. demostró que las intervenciones educativas en el cuidado de la diabetes contribuyen a mejorar el control metabólico de la enfermedad, 
incrementar el conocimiento sobre la diabetes y reducir la necesidad de medicación de los enfer$\operatorname{mos}^{22}$; la evidencia científica es tan concluyente que todas las guías clínicas desarrolladas en el área de la diabetes mellitus establecen explícitamente que la base del control de la enfermedad es la educación del paciente y la de su grupo familiar: Federación Internacional de Diabetes ${ }^{23}$, National Institut for Health and Clinical Excellence ${ }^{24}$, Asociación Americana de Diabetes ${ }^{25}$, Ministerio de Salud Chile -guías clínicas diabetes mellitus tipo $2{ }^{26}$. En Chile, la diabetes mellitus como problema de salud está incorporada en el Régimen de Garantías en Salud (GES), por lo cual, todos los pacientes afectados por esta patología tienen garantizada su atención ${ }^{27}$. En nuestro país, no encontramos publicaciones relativas a programas educativos estructurados para enfermos diabéticos (esto es: que posean un manual para el educador que describa los contenidos, la metodología a utilizar en la capacitación y los tiempos asignados a cada actividad) que hayan evaluado científicamente su eficacia; normalmente, la educación que se realiza obedece a iniciativas locales no estructuradas, las cuales no disponen de los elementos que caracterizan a los programas estructurados descritos y, por consiguiente, carecen de respaldo científico respecto a si realmente contribuyen a mejorar el control metabólico de los enfermos.

El presente trabajo fue realizado con el propósito de evaluar la eficacia de un programa educativo estructurado, específicamente diseñado para la población diabética chilena. En la elaboración del programa educativo, así como en la validación del mismo, participaron profesionales pertenecientes al Servicio de Salud del Maule, a la Universidad Católica del Maule, a la Clínica Alemana de Santiago, a la Asociación de Diabéticos de Chile de Santiago (ADICH) y al Departamento de Salud Municipal de Talca.

\section{Material y Métodos}

\section{Estructura y contenidos del programa educativo}

El programa educativo para pacientes diabéticos aborda los aspectos cognitivos, emocionales y vivenciales relativos a la enfermedad y está estructurado en 6 unidades básicas y 2 unidades complementarias. Las 6 unidades básicas son: 1) Generalidades de la diabetes mellitus y aceptación de la enfermedad; 2) Complicaciones crónicas; 3) Complicaciones agudas y autocontrol; 4) Nutrición y alimentación; 5) Control de la enfermedad; 6) Viviendo con diabetes (elaboración de una rutina cotidiana para vivir con diabetes); las unidades complementarias son: 7) Insulinoterapia; 8) Embarazo y diabetes mellitus. El programa se desarrolla en una modalidad de taller en grupos de 8 pacientes, dirigido por un educador específicamente entrenado para estos efectos; cada módulo o sesión tiene una duración de 90 minutos y estas se deben realizar una vez por semana. El educador tiene a su disposición un manual que detalla el contenido y la metodología de cada unidad; además, dispone de material audiovisual y material educativo de apoyo para cada módulo.

\section{Validación del instrumento}

Previo a la evaluación científica del programa educativo, este fue validado en un grupo focal de 8 personas, todos los sujetos que participaron en este proceso eran portadores de diabetes mellitus tipo 2. El resultado global de la validación demostró que el programa se ajustaba plenamente a su diseño teórico y los pacientes expresaron un alto grado de satisfacción al finalizar su participación en la actividad educativa.

\section{Evaluación científica}

Para evaluar la eficacia del programa educativo se realizó un ensayo clínico aleatorio de 2 ramas (grupos); el grupo A (experimental) asistió a sus controles habituales y, además, los enfermos asistieron a las 6 unidades básicas del programa educativo estructurado; la capacitación se realizó una vez a la semana durante 6 semanas; el grupo $\mathrm{B}$ (control) recibió las prestaciones que regularmente se realiza a los pacientes diabéticos en la institución, en la cual controlan su problema de salud; esta actividad incluye capacitación individual no estructurada que responde a las necesidades clínicas específicas del paciente en el momento de la consulta. Los sujetos incorporados en el estudio fueron pacientes diabéticos en control en establecimientos de atención primaria del sistema público de salud que cumplían con los criterios de inclusión definidos para la investigación. La asignación de los pacientes a las diferentes ramas o grupos se realizó al azar. La investigación fue aprobada por el Comité de Ética Científica del Servicio de Salud del Maule y, todos los pacien- 
tes participantes firmaron un consentimiento informado.

El tamaño muestral del grupo a estudiar fue calculado considerando un nivel de confianza de $95 \%$, una potencia de la prueba de $80 \%$, y la menor diferencia observada en estudios similares en los niveles de hemoglobina glicosilada entre el grupo intervenido y aquel sin intervención $(0,8)$; el tamaño muestral así calculado fue incrementado en $10 \%$ por la posible pérdida de pacientes en el seguimiento.

La intervención educativa fue realizada por 7 enfermeras en grupos de 8 pacientes, 6 enfermeras capacitaron a un grupo y 1 enfermera capacitó a dos grupos, la misma profesional que realizaba la intervención era la responsable del seguimiento de los pacientes del grupo experimental y del grupo control. Los pacientes del grupo intervenido que no completaron las 6 sesiones del programa educativo no fueron sustituidos, así como los pacientes del grupo intervenido y control que no completaron los 12 meses de seguimiento. En definitiva, se estudiaron 115 pacientes; 59 pacientes fueron asignados al grupo experimental y 56 al grupo control.

\section{Criterios de inclusión}

Los criterios de inclusión considerados para ambos grupos de pacientes fueron:

Ser portador de una diabetes mellitus tipo 2, edad menor de 65 años, menos de 10 años de evolución de la enfermedad desde el diagnóstico, instruido, con una hemoglobina glicosilada igual o mayor de 7,5\%, personas autónomas mental y físicamente,tratamiento con hipoglicemiantes orales, insulina o ambos.

\section{Criterios de exclusión}

Los criterios de exclusión fueron: analfabetismo, demencia, deterioro cognitivo o enfermedad psiquiátrica severa, incapacidad para desarrollar las actividades básicas cotidianas, estar afectado por alguna complicación crónica mayor de la diabetes, esto es: ceguera, enfermedad coronaria con insuficiencia cardiaca, insuficiencia renal crónica avanzada (etapa IV o más), amputación mayor en algunas de sus extremidades inferiores.

\section{Estudio y análisis}

La investigación se realizó entre enero del año 2014 y diciembre del 2015 en establecimientos de atención primaria pertenecientes a la Red de Salud Pública de la Región del Maule. La intervención (programa educativo) se realizó en grupos de 8 pacientes por siete enfermeras entrenadas en el programa educativo. Ambos grupos (experimental y control)fueron seguidos por un período de 12 meses, a todas las personas se les realizó un control al inicio de la investigación y, posteriormente, cada 4 meses; en cada control se registró el índice de masa corporal, presión arterial, hemoglobina glicosilada y perfil lipídico. A todos los pacientes se les realizó un electrocardiograma, examen de fondo de ojo, creatininemia y microalbuminuria. Los parámetros a estudiar fueron recolectados en un instrumento específicamente diseñado para estos efectos. Para el análisis estadístico se utilizó la prueba de normalidad de Kelmogorov-Smirnov, la prueba de homogeneidad de varianza de Levene y la prueba de $t$ de comparación de medias para muestras independientes y muestras relacionadas, para realizar los cálculos estadísticos se utilizó el software SPSS versión 21.

\section{Resultados}

\section{Características socio-demográficas}

En la Tabla 1 se presenta la distribución por sexo de los pacientes que participaron en la investigación. La edad promedio fue 53,14 años (DS 8,99) en el grupo experimental y 50,55 años (DS 9,55) en el grupo control. El 59\% de los pacientes tenía escolaridad básica ( $57 \%$ en el grupo experimental y $61 \%$ el grupo control), sólo $8,6 \%$ del grupo investigado tenía estudios técnicos o universitarios (2,4\% en el grupo experimental y $15,4 \%$ en el grupo control).

\section{Control metabólico}

\section{Análisis global}

En ambos grupos hubo una reducción de los niveles de hemoglobina glicosilada en las 3 mediciones realizadas posteriores al ingreso, en todos los registros, la reducción fue mayor en el grupo experimental respecto al grupo control. Las mayores diferencias en la reducción del valor de hemoglobina glicosilada del grupo experimental respecto al grupo control se observaron entre el ingreso y la medición correspondiente al mes 4 $(0,4 \%)$ y entre el ingreso y la medición realizada en el mes $8(0,46 \%)$ (Tabla 2$)$. 
Análisis por educadora

En el grupo experimental, el nivel de reducción de los valores de hemoglobina glicosilada fue distinto en las diferentes profesionales que realizaron la intervención educativa; en el caso de las educadoras 1,5 y 7 , se observó una reducción mayor a 1,4\% entre el ingreso y la medición del mes 12 ( $\mathrm{p}<0,05)$. En el grupo control, ninguna de las diferencias observadas entre el ingreso y el mes 12 resultó estadísticamente significativa (Tablas 3 y 4 ).

Tabla 1. Evaluación Programa Educativo Estructurado Diabetes. Población según sexo y tipo de intervención

\begin{tabular}{|c|c|c|c|c|c|c|}
\hline \multirow[t]{3}{*}{ Sexo } & \multicolumn{4}{|c|}{ Grupo } & \multicolumn{2}{|c|}{ Total } \\
\hline & \multicolumn{2}{|c|}{ Experimental } & \multicolumn{2}{|c|}{ Control } & & \\
\hline & $\mathbf{n}$ & $\%$ & $\mathbf{n}$ & $\%$ & $\mathbf{n}$ & $\%$ \\
\hline Masculino & 10 & 17 & 20 & 36 & 30 & 26,1 \\
\hline Femenino & 49 & 83 & 36 & 64 & 85 & 73,9 \\
\hline Total & 59 & 100 & 56 & 100 & 115 & 100 \\
\hline
\end{tabular}

Tabla 2. Control metabólico. Grupo Experimental - Grupo Control

\begin{tabular}{|lcccccc|}
\hline Grupo & Ingreso & $\begin{array}{c}\text { Hemoglobina glicosilada }(\overline{\mathbf{x}}) \\
\text { Mes 4 }\end{array}$ & Mes 8 & Mes 12 & Diferencia & ingreso- mes 12 \\
Experimental & 10,05 & 9,12 & 8,99 & 9,11 & 0,94 & $<0,05$ \\
Control & 9,86 & 9,33 & 9,26 & 9,25 & 0,61 & $<0,05$ \\
\hline
\end{tabular}

Tabla 3. Control metabólico por educadora. Grupo experimental

\begin{tabular}{|c|c|c|c|c|c|c|c|}
\hline \multirow[t]{2}{*}{ Educadora } & \multirow[t]{2}{*}{ n pacientes } & \multicolumn{4}{|c|}{ Hemoglobina glicosilada $(\bar{x})$} & \multirow{2}{*}{$\begin{array}{c}\text { Diferencia } \\
\text { ingreso - mes } 12\end{array}$} & \multirow[t]{2}{*}{ p* } \\
\hline & & Ingreso & Mes 4 & Mes 8 & Mes 12 & & \\
\hline 1 & 8 & 10,56 & 7,61 & 8,03 & 8,30 & 2,26 & $<0,05$ \\
\hline 2 & 15 & 9,59 & 9,25 & 8,83 & 9,20 & 0,39 & $<0,05$ \\
\hline 3 & 8 & 9,83 & 9,60 & 9,40 & 9,61 & 0,22 & N.S. \\
\hline 4 & 6 & 9,98 & 10,31 & 10,28 & 10,17 & $-0,19$ & N.S. \\
\hline 5 & 7 & 10,63 & 8,41 & 8,19 & 8,88 & 1,75 & $<0,05$ \\
\hline 6 & 7 & 10,10 & 9,79 & 9,00 & 9,15 & 0,95 & N.S. \\
\hline 7 & 8 & 10,11 & 9,04 & 9,62 & 8,62 & 1,49 & $<0,05$ \\
\hline
\end{tabular}

*N.S. $=$ No significativo.

Tabla 4. Control metabólico por educadora. Grupo Control

\begin{tabular}{|c|c|c|c|c|c|c|c|}
\hline \multirow[t]{2}{*}{ Educadora } & \multirow[t]{2}{*}{ n pacientes } & \multicolumn{4}{|c|}{ Hemoglobina glicosilada $(\bar{x})$} & \multirow{2}{*}{$\begin{array}{c}\text { Diferencia } \\
\text { ingreso - mes } 12\end{array}$} & \multirow[t]{2}{*}{$\mathbf{p}^{*}$} \\
\hline & & Ingreso & Mes 4 & Mes 8 & Mes 12 & & \\
\hline 1 & 8 & 10,90 & 9,56 & 9,86 & 9,89 & 1,01 & N.S. \\
\hline 2 & 13 & 9,50 & 9,08 & 9,02 & 9,40 & 0,01 & N.S. \\
\hline 3 & 8 & 9,00 & 9,36 & 9,46 & 9,28 & $-0,28$ & N.S. \\
\hline 4 & 8 & 9,55 & 9,17 & 8,86 & 8,66 & 0,89 & N.S. \\
\hline 5 & 7 & 9,46 & 8,83 & 8,70 & 8,04 & 1,42 & N.S. \\
\hline 6 & 3 & 9,00 & 10,00 & 9,90 & 9,10 & $-0,10$ & N.S. \\
\hline 7 & 9 & 11,09 & 9,74 & 9,48 & 9,97 & 1,12 & N.S. \\
\hline
\end{tabular}

*N.S. = No significativo. 
Tabla 5. Evaluación parámetros clínicos. Programa Educativo Estructurado Diabetes

\begin{tabular}{|c|c|c|c|c|c|c|}
\hline & \multicolumn{3}{|c|}{ Grupo Experimental } & \multicolumn{3}{|c|}{ Grupo control } \\
\hline & Ingreso $(\bar{x})$ & Mes $12(\bar{x})$ & $\mathbf{p}$ & 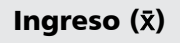 & Mes $12(\bar{x})$ & $\mathbf{p}$ \\
\hline Índice masa corporal & 33,01 & 32,99 & N.S. & 32,05 & 32,42 & N.S. \\
\hline Perímetro abdominal $(\mathrm{cm})$ & 104,25 & 104,44 & N.S. & 102,07 & 104,45 & N.S. \\
\hline Presión sistólica (mmHg) & 135,98 & 134,97 & N.S. & 132,36 & 134,35 & N.S. \\
\hline Presión diastólica (mmHg) & 76,07 & 77,47 & N.S. & 78,30 & 79,40 & N.S. \\
\hline Colesterol total (mg/dL) & 185,77 & 187,29 & N.S. & 183,86 & 190,15 & N.S. \\
\hline Colesterol LDL (mg/dL) & 101,23 & 102,06 & N.S. & 104,64 & 104,94 & N.S. \\
\hline Colesterol HDL (mg/dL) & 45,48 & 43,69 & N.S. & 46,04 & 45,84 & N.S. \\
\hline Triglicéridos (mg/dl) & 190,23 & 178,24 & N.S. & 190,58 & 195,16 & N.S. \\
\hline
\end{tabular}

${ }^{*}$ N.S. $=$ No Significativo.

\section{Parámetros clínicos}

El índice de masa corporal y el perímetro abdominal no registraron variaciones significativas en el grupo experimental ni en el grupo control durante el período de observación. Del mismo modo, la presión arterial sistólica y diastólica, el colesterol total, LDL y HDL y triglicéridos no registraron variaciones significativas en ninguno de los dos grupos estudiados durante los 12 meses de investigación (Tabla 5).

\section{Discusión}

Los trabajos científicos que han estudiado el tema de la educación en diabetes mellitus han demostrado sistemáticamente que los programas educativos estructurados representan un pilar fundamental en el control de la enfermedad, el hecho que el diabético asuma un rol protagónico en el cuidado de su afección, no sólo favorece el control metabólico de la misma, sino que también incrementa la adherencia del paciente a sus controles, contribuyendo a mejorar su estado nutricional y los parámetros clínicos relacionados con su enfermedad; la sumatoria de estos beneficios producen una mejoría en la calidad de vida $y$, consecuentemente, en el largo plazo reducen la probabilidad que se desarrollen las complicaciones propias de la diabetes mellitus s.-22 $^{\text {. }}$

En el presente trabajo, la reducción del nivel de hemoglobina glicosilada observada en un horizonte de 12 meses en el grupo que participó en el pro- grama educativo estructurado fue $35 \%$ superior al grupo control; la mejoría verificada en el grupo experimental $(0,94 \%)$ estuvo dentro del rango reportado en los trabajos científicos extranjeros que han estudiado el impacto de la educación en diabetes mellitus ${ }^{5,6,14-18}$. La equivalencia del logro obtenido respecto a la experiencia internacional indica que el instrumento evaluado es comparable en eficacia con los programas educativos estructurados elaborados en otros países y responde a las necesidades de la población diabética chilena.

El análisis de las otras variables clínicas incluidas en la investigación demostró que los pacientes al ingreso presentaban un adecuado control de su presión arterial, tanto sistólica como diastólica, asimismo en los registros correspondientes a colesterol total, colesterol LDL y HDL. Las cifras dan cuenta que los enfermos cumplían las metas de control sugeridas por la Asociación Americana de Diabetes ${ }^{25}$; estos parámetros no experimentaron variaciones durante la investigación, por lo cual, si bien no pudo evidenciarse una mejoría de los mismos, si se puede señalar que la educación estructurada y no estructurada contribuyeron a mantener estas variables clínicas controladas. Una situación distinta se observó en las mediciones correspondientes al índice de masa corporal y al perímetro abdominal; en estos últimos, las cifras de ingreso a la investigación dieron cuenta que los valores estaban por sobre lo recomendado, manteniéndose sin modificaciones durante todo el estudio. El hecho que los pacientes que presentaban sobrepeso u obesidad no hayan registrado 
una mejoría de su estado nutricional después del programa educativo indica que para lograr una reducción de peso sería recomendable incorporar intervenciones específicas para este objetivo.

Por otra parte, el hecho que en la presente investigación, en el grupo experimental se haya observado distintos niveles de mejoría del control metabólico dependiendo de la educadora que realizó la capacitación, establece la necesidad de realizar estudios que permitan identificar las variables que expliquen estas diferencias, para precisar los factores que inciden en ello.

Adicionalmente, es importante señalar que toda intervención educativa dirigida a pacientes portadores de una diabetes mellitus debería realizarse dentro de un contexto que incorpore a la familia, los amigos y las organizaciones de la comunidad en la cual él vive, idealmente en el marco de una política gubernamental que garantice la atención de los pacientes afectados por una enfermedad crónica, posibilitando que el paciente posea una red social que lo apoye, en un ambiente que le facilite mantener un adecuado control de su problema de salud ${ }^{28,29}$.

Finalmente, se debe señalar que la introducción de programas educativos estructurados eficaces para paciente diabéticos, hoy en día constituye una obligación para cualquier organización o profesional dedicado a la atención de pacientes afectados por este problema de salud, dada la fuerte evidencia científica que avala esta recomendación; en este sentido, el programa educativo evaluado por los autores constituye una alternativa válida disponible para el sistema de salud chileno.

\section{Referencias}

1. Encuesta Nacional de Salud ENS Chile 2009-2010.

2. Uk Prospective Diabetes Study (UKPDS) Group. Intensive Blood-glucose control with sulphonylureas or insulin compared with conventional treatment and risk of complications in patients with type 2 diabetes (UKPDS 33). Lancet 1998; 352: 837-53.

3. The Diabetes Control and Complications Trial Research Group. The effect of intensive treatment of diabetes on the development and progression of long-term complications in insulin-dependent diabetes mellitus. N Engl J Med 1993; 329: 977-86.

4. Duncan I, Birkmeyer C, Coughlin S, Li QE, Sherr D, Boren S. Assessing the value of diabetes education. Diabetes Educ 2009; 35: 752-60.
5. Norris SL, Lau J, Smith SJ, Schmid CH, Engelgau MM. Self-management education for adults with type 2 diabetes: a meta-analysis of the effect on glycemic control. Diabetes Care 2002; 25: 1159-71.

6. Gary TL, Genkinger JM, Guallar E, Peyrot M, Brancati FL. Meta-analysis of randomized educational and behavioral interventions in type 2 diabetes. Diabetes Educ 2003; 29: 488-501.

7. Renders CM, Valk GD, Griffin SJ, Wagner EH, EijK Van JT, Assendelft WJ. Interventions to improve the management of diabetes in primary care, outpatient, and community settings: a systematic review. Diabetes Care 2001; 24: 1821-33.

8. Peyrot M, Rubin RR, Funnell MM, Seminerio LM. Acces to diabetes selfmanagement education: results of national surveys of patients, educators, and physicians. Diabetes Educ 2009; 35: 246-8, 252-6, 258-63.

9. Funnell MM, Nwankwo R, Gillard ML, Anderson RM, Tang TS. Implementing an empowerment-based diabetes selfmanagement education program. Diabetes Educ 2005; 31: 53, 55-56, 61.

10. Martin AL. Changes and consistencies in diabetes education over 5 years: results of the 2010 national Diabetes Education Practice Survey. Diabetes Educ 2012; 38: 3546.

11. Siminerio LM, Piatt G, Zgibor JC. Implementing the chronic care model for improvements in diabetes care and education in a rural primary care practice. Diabetes Educ 2005; 31: 225-34.

12. Boren SA, Fitzner KA, Panhalkar PS, Specker JE. Costs and benefits associated with diabetes education: a review of the literature. Diabetes Educ 2009; 35: 72-96.

13. Duke SA, Colagiuri S, Colagiuri R. Individual patient education for people with type 2 diabetes mellitus. Cochrane Database Syst Rev 2009; (1): CD005268.

14. Piatt GA, Anderson RM, Brooks MM, Sanger T, Siminerio LM, Korytkowski MM, et al. 3-Year follow-up of clinical and behavioral improvements following a multifaceted diabetes care intervention: results of a randomized controlled trial. Diabetes Educa 2010; 36 (2): 301-9.

15. Ko SH, Song KH, Kim SR, Lee JM, Kim J-S, Shin J-H, et al. Long-term effects of a structured intensive diabetes education programme (SIDEP) in patients with type 2 diabetes mellitus-a 4-year follow-up study. Diabet Med 2007; 24 (1): 55-62.

16. Trento M, Passera P, Tomalino M, Bajardi M, Pomero F, Allione A, et al. Group visits improve metabolic control in type 2 diabetes: a 2-year follow-up. Diabetes Care 2001; 24: 995-1000.

17. Trento M, Passera P, Borgo E, Tomalino M, Bajardi M, 
Cavallo F, et al. A 5-year randomized controlled study of learning, problem solving ability, and quality of life modifications in people with type 2 diabetes managed by group care. Diabetes Care 2004; 27 (3): 670-5.

18. Steinsbekk A, Rygg L $\varnothing$, Lisulo M, Rise MB, Fretheim A. Group based diabetes self-management education compared to routine treatment for people with type 2 diabetes mellitus. A systematic review with meta-analysis. BMC Health Serv Res 2012; 12: 213.

19. Powers MA, Bardsley J, Cypress M, Duker P, Funnell MM, Hess Fischl A, et al. Diabetes self-management education and support in type 2 diabetes: a joint position statement of the American Diabetes Association, the American Association of Diabetes Educators, and the Academy of Nutrition and Dietetics. Diabetes Care 2015; 38: 1372-82.

20. Cochran J, Conn VS. Meta-analysis of quality of life outcomes following diabetes self-management training. Diabetes Educ 2008; 34 (5): 815-23.

21. Baradarn HR, Shams-Hosseini N, Noori-Hekmat S, Tehrani-Banihashemi A, Khamseh ME. Effectiveness of diabetes educational interventions in Iran: a systematic review.Diabetes Technol Ther 2010; 12 (4): 317-31.

22. Deakin T, McShance CE, Cade JE, Williams RD. Group based training for self-management strategies in people with type 2 diabetes mellitus. Cochcrane Database Syst
Rev 2005; (2): CD003417.

23. International Diabetes Federation Clinical Guidelines. Task Face. Globalguidelines for type 2 diabetes. Brusseles, Belgium International Diabetes Federation 2012.

24. National Institute for Health and Care Excellence. Guidance Type 2 Diabetes in Adults disponible en www.nice.org.uk/guidance/conditions-and-diseases/ diabetes-and-other-endocrinol-nutritional-and-metabolic-conditions/diabetes.

25. American Diabetes Association. Standards of medicalcare in diabetes 2016. Diabetes Care, January 2016, volume 39, supplement 1 .

26. Guía Clínica Diabetes mellitus tipo 2. Ministerio de Salud. Santiago Minsal, 2010.

27. Ministerio de Salud de Chile. Decreto Supremo $N^{\circ} 03$ del 27 de enero del 2016. Aprueba Garantías Explícitas en Salud del Régimen General de Garantías en Salud.

28. Marrero DG, Ard J, Delamater AM, Peragallo-Dittko V, Mayer-Davis EJ, Nwankwo R, et al. Twenty-first century behavioral medicine: a context for empowering clinicians and patients with diabetes: a consensus report. Diabetes Care 2013; 36: 463-70.

29. Fisher EB, Brownson CA, O’Toole ML, Shetty G, Anwuri VV, Glasgow RE. Ecological approaches to self-management: the case of diabetes. Am J Public Health 2005; 95: 1523-35. 